\title{
The directionality of interjoint neural coupling in the impaired upper extremity post stroke
}

This paper was downloaded from TechRxiv (https://www.techrxiv.org).

\section{LICENSE}

CC BY 4.0

SUBMISSION DATE / POSTED DATE

06-01-2022 / 17-01-2022

\section{CITATION}

Kim, Dongwon; Koh, Kyung; Baghi, Raziyeh; Lo, Li-Chaun; Zhang, Chunyang; Zhang, Li-Qun (2022): The directionality of interjoint neural coupling in the impaired upper extremity post stroke. TechRxiv. Preprint. https://doi.org/10.36227/techrxiv.17912558.v1

$\mathrm{DOI}$

10.36227/techrxiv.17912558.v1 


\title{
The directionality of interjoint neural coupling in the impaired upper extremity post stroke
}

\author{
Abbreviated title: Directionality of stroke-caused coupling \\ Dongwon Kim ${ }^{1}$, PhD, Kyung Koh ${ }^{2}$, PhD, Raziyeh Baghi ${ }^{2}$, MS, Li-Chuan Lo ${ }^{2}$, PhD, Chunyang \\ Zhang $^{2}$, MD, Giovanni Oppizzi ${ }^{2}$, BS, and Li-Qun Zhang ${ }^{2-4}, \mathrm{PhD}$ \\ ${ }^{1}$ EpicWide, LLC, ${ }^{2}$ Department of Physical Therapy and Rehabilitation Science, University of Maryland, \\ Baltimore, MD, USA, ${ }^{3}$ Department of Orthopedics, University of Maryland, Baltimore, MD, USA, \\ ${ }^{4}$ Department of Bioengineering, School of Engineering, University of Maryland, College Park, MD, USA
}

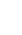

10

1

12

3

4

15

2 Keywords: Stroke, neural coupling, coupling directionality, abnormal coordination, robotic 3 rehabilitation 


\section{Abstract}

25

1

2

3

6

4

9

0
To date, research has primarily focused on identifying and analyzing abnormal coupling in the upper extremity following stroke during gross movements such as reaching. Yet, little is known about the direction of abnormal coupling in conjunction with its degree between jointsmuscles - of interest. In this study, we address the directionality of pathological neural couplings between joints, and variability in the degree of them, in the upper extremity post stroke. To evaluate the direction of interjoint coupling, joint motion and muscle activity in an involuntary mode while another joint-muscle - was voluntarily activated were assessed in comparison to those made in a volitional mode, focusing on the shoulder, elbow and wrist joints. Overall, involuntary activation of the wrist flexor in the stroke group is observed when muscles of a more proximal joint voluntarily activate $(\mathrm{p}<0.1$ and $\mathrm{p}<0.05)$, while activation of the wrist flexor does not lead to involuntary activation of the shoulder muscles and elbow flexor, in comparison to the healthy group. In particular, in the stroke group who is assumed to have a severe loss of the corticospinal system, the wrist extensor is subordinately activated by those about the other joints voluntarily activated $(\mathrm{p}<0.05)$ while it does not cause activations of those about the other joints. These findings indicate that stroke-caused synergies could be directional, implying the existence of a cortical source and/or neural pathway for a muscle that is not paired with that for other muscles.

4

5

8


52 Stroke can be characterized by impaired motor coordination as it evolves to the stage following 53 the acute phase(Beer, Dewald and Rymer, 2000; Wilkins et al., 2017). To achieve a smooth 54 manipulation, each joint of the arm should fulfill its allocation for the movement in a harmonic manner according to the control plan orchestrated by the central nervous system (CNS)(Beer, Dewald and Rymer, 2000; Cirstea and Levin, 2000). Damaged joint individuation not only 57 deprives individuals post stroke of dexterous manipulation, but also stereotypes motoric movement.

A loss of independent joint control perhaps originates partially or mostly from abnormal coupling between joints. Indeed, studies have provided evidence that pathological patterns of muscle coactivation appear in individuals with stroke(Cheung, D’Avella and Bizzi, 2009; Roh et al., 2013).

Stroke causes a lesion at different sites over cortical areas and neural pathways between the cortex and motor neuron pools(McMorland, Runnalls and Byblow, 2015). According to the lesioned region, the severity of impairment could vary across joints of the upper limb. The corticospinal system has distributed and inter-tangled connections to motor neuron pools, each of which controls more proximal or more distal muscles, respectively. In the cortical surface, motor areas that are linked with proximal and distal joints significantly overlap(Fetz and Cheney, 1980; Schieber, 2001). Even in the subcortical structures, axons descending from cortical areas for proximal and distal muscles are bundled together(Fetz and Cheney, 1980). Due probably to these facts, we have observed inconclusive results of stroke regarding the severity gradient of motor deficits across the impaired arm(Colebatch and Gandevia, 1989; Beebe and Lang, 2008).

Though it is obvious that neural couplings between the joints of the upper extremity (through their related muscles) after stroke exist during manipulation(Beer, Dewald and Rymer, 2000; Ellis et al., 2016; Owen, Ingo and Dewald, 2017; Barry et al., 2021), special attention has not yet been paid to the direction of coupling in conjunction with the degree of coupling. Though several researchers reported the directionality of coupling between the brain and the muscle(Wickens, Hyland and Anson, 1994; Yang et al., 2018), little known is about the directionality of strokecaused abnormal coupling between joints - muscles - in the upper extremity. It is possible that in a pair of joints, one joint moves in an involuntarily manner when voluntarily moving the other joint, but the other joint does not move when volitionally moving the joint. Likewise, it is possible that in a pair of muscles, one muscle is activated in an involuntarily manner when the other muscle 
81 is activated, but the other muscle is not activated when the muscle is activated. These phenomena 82 would imply the existence of a cortical source and/or neural pathway for the joint or muscle that is not paired with that for the other joint or muscle. It is worth investigating the direction of coupling as well as the severity of coupling between joints of a stroke-affected arm to gain an insight into diffuse projection of cortical sources and routes that convey neural drive to a certain muscle or joint.

87 In this study, we investigate the direction of coupling as well as the extent of coupling between joints and between muscles of the upper limb of a population with moderate-to-severe impairment post stroke. Couplings between the shoulder, elbow and wrist of the impaired arm in individuals with stroke are evaluated. Motions at the joints of the arm caused by voluntary motion at a target individual joint due to stroke-induced couplings are quantified when moving the target joint while keeping the non-target joints stationary. The existence of no coupling or unidirectional coupling that would imply the existence of a separate cortical region and connections for controlling the joint is examined.

We strive to distinguish the features of stroke-caused coupling based on the severity of corticospinal system damage. Originally stroke damages the corticospinal system, leading to use of the reticulospinal tract (RST), as a substitute of the corticospinal tract (CST)(Baker, 2011; Wilkins et al., 2017; Senesh, Barragan and Reinkensmeyer, 2020). Stroke survivors who are assumed to use the RST as the main tract that conveys motor drives typically show increased synergetic movements and decreased controllability of distal joints(Turton et al., 1996), whereas stroke survivors who are assumed to use the CST as the main tract with the ability to control individual muscles(Baker, 2011; Senesh, Barragan and Reinkensmeyer, 2020). We examine whether the main tract in use affects the direction and extent of stroke-caused coupling.

\section{METHODS}

\section{Participants}

A total of 14 hemiplegic volunteers post stroke (age: $53.86 \pm 14.68$ (S.D) years; sex (F/M): 3/11; 108 impaired side (L/R): 6/8, time since stroke: $6.75 \pm 4.15$ (S.D) month) participated in the study. The 109 inclusion criteria were moderate-to-severe upper extremity impairment (upper extremity Fugl- 
Meyer (UEFM) <50) and sufficient cognitive/language abilities to follow instructions during the experiment (Mini-Mental Status Score $>22$ ). We excluded volunteers who had severe shoulder pain, relevant musculoskeletal injury, or fixed contraction deformity in the upper extremity. None of the participants received pharmacological medications for spasticity and tone (i.e. Botulinum toxin injection to the upper limb) in the 5 months before the experiment. A total of 8 volunteers with no neurological or motor deficit (age: $51.25 \pm 14.50$ years; $\operatorname{sex}(\mathrm{F} / \mathrm{M})$ : $5 / 3$ ) were also recruited. Each participant gave written informed consent approved by the Institutional Review Board of the University of Maryland, Baltimore.

\section{Apparatus}

An upper-limb exoskeleton robot capable of controlling the shoulder (SHL), elbow (ELB) and wrist (WRS) joints in an individual or simultaneous manner on the horizontal plane18 was used to control and measure the motion of each joint. Motor control and data acquisition were performed in a LabVIEW environment. The impedance of the individual joint of the exoskeleton was minimized under impedance control. Six wireless pre-amplified, differential surface EMG electrodes (Trigno, Delsys Inc.) were used to acquire EMG signals from the anterior deltoid (AD), posterior deltoid (PD), biceps brachii (Bi), triceps brachii (Tri), flexor carpi radialis (FCR), and extensor carpi radialis (ECR) muscles19. Data acquisition of EMG signals were conducted in a LabVIEW environment. The sampling rate was set at $1000 \mathrm{~Hz}$.

\section{Procedure}

Participants sat upright comfortably on a height-adjustable chair, placing their arm on the exoskeleton. The segment lengths of the exoskeleton for the upper arm and forearm were then adjusted, respectively, to align mechanical axes for shoulder horizontal adduction/abduction, and elbow and wrist flexion/extension with the anatomical joint axes of the participants. The upper arm and forearm were strapped, with braces, to ensure good alignment throughout the experiment. The initial configuration of the arm was at shoulder adduction of $45^{\circ}$, shoulder flexion of $45^{\circ}$, shoulder horizontal adduction of $70^{\circ}$, elbow flexion of $60^{\circ}$, and wrist flexion of $0^{\circ}$, respectively.

The experiment consisted of three sessions. Participants were asked to voluntarily move only one joint (the target joint) with the other joints (non-target joints) resting, as depicted in Fig. 1. The shoulder, elbow and wrist joints of the exoskeleton were able to be moved freely with minimized 
mechanical impedance. The target joint was selected in the order of the shoulder, elbow and wrist joints. The movement and its speed at each joint were designed as 7 9 cycles at self-selected comfortable speeds. Break times were inserted to mitigate the fatigue effect during the experiment.

\section{Data processing}

The angle and EMG data were time synchronized using a pulse signal sent to different personal computers used during the data acquisition. Raw angle data were used themselves without filtering. Raw EMG data were high-pass filtered at $20 \mathrm{~Hz}$, de-meaned, rectified, and low-pass filtered at 5 $\mathrm{Hz}$ to get the EMG linear envelopes (LEs). The mean baseline EMG LE of each muscle for each session was acquired while the participant rested and was subtracted from the EMG LE data of the corresponding muscle for the same session. For analyzing measures explained below, analyzing windows for each session of movement and each movement direction were selected from the starting point of an inward or outward movement of the target joint to its stopping point for each cycle. The starting and stopping points were located at the zero velocity of the joint. The first inward or outward movement from the initial position of each session was excluded from analysis.

\section{Measures}

The basic measure of joint motion coupling was the ratio of the extent of involuntary motion versus that of voluntary motion at a joint of interest. The ratio provided an insight into how much involuntary motion at a non-joint of interest was made when the target joint moved with reference to the extent of motion at the non-joint when the joint was the target joint. We used the absolute values of the angular displacements of each joint to quantify the extent of coupling between two joints. In a similar way, the basic measure of muscle activation was the ratio of the extent of involuntary activation versus that of voluntary activation at a muscle of interest. The averaged values across the selected cycles were used for the ratios. A ratio of 0 indicates that the joint or muscle of interest is entirely decoupled when another joint voluntarily moves or another muscle is voluntarily activated, while a ratio of 1 indicates the same amount of joint motion or muscle activation is produced in the involuntary mode in comparison to that produced in the voluntary mode. Ratios beyond 1 imply that the amount of joint motion or muscle activation produced in the involuntary mode is greater than that produced in the voluntary mode. We saturated the values of ratios beyond 2 to 2 to reduce the variability of ratios. We separated motions when the segments of the arm moved inward from those made when the segments of the arm moved outward. 


\section{Analysis}

170 We employed multivariate analysis of variance (MANOVA) with Bonferroni corrections to 171 investigate differences between groups in ratios for motion coupling and muscle coupling. For 172 motion coupling, two-way MANOVA was performed on the six ratios, with group as a between173 subject variable and with direction as a within-subject variable. For muscle coupling, one-way 174 MANOVA was performed on the 24 ratios with group as a between-subject variable. We used 175 one-tailed t-tests to determine whether ratios were significantly lower than 1.

176 Correlation analyses were conducted to evaluate the relationships of the UEFM score with the 177 maximum angular displacements during voluntary flexion or extension of the shoulder, elbow and 178 wrist, respectively. We aimed at identifying stroke participants who are assumed to employ the 179 RST versus the CST through correlation analyses.

180 Statistical analyses were performed with SPSS (Windows v.21, SPSS Inc.). Significance level 181 was set at 0.05 .

\section{Data availability}

183 The data that support the findings of this study are available from the corresponding author on 184 reasonable request.

\section{RESULTS}

187 Mainly, we found that the wrist flexor tends to involuntarily activate (marginally) significantly 188 more in the stroke group when the shoulder or elbow voluntarily moves, in comparison to the 189 healthy group. Meanwhile, it is interesting that activation of the wrist flexor does not lead to 190 involuntary activation of $\mathrm{AD}, \mathrm{PD}$ and $\mathrm{Bi}$, which would imply that abnormal coupling is directional. 191 In the stroke group who is assumed to be with a severe loss of the corticospinal system, the wrist 192 extensor is subordinately activated by those about other joints voluntarily activated, while it does 193 not cause activations of those about other joints. Fig. 2 shows the results of a representative 194 individual post stroke.

\section{1. Results on the whole stroke participants}


MANOVA reveals no main effect of group in ratios for joint motion coupling ( $\mathrm{p}>0.1)$. We observe no effects of movement direction $(p>0.9)$ and interaction $(p>0.9)$, indicating no significant differences in the extent of motion coupling between inward movements and outward movements (refer to Fig. 3(a)). However pairwise comparisons between groups report that the wrist joint in the stroke group tends to involuntarily move greater when the shoulder $(\mathrm{p}=0.051)$ or elbow $(p=0.058)$ voluntarily moves and that the shoulder $(p=0.064)$ and elbow joint $(p=$ 0.003 ) in the stroke group involuntarily move greater when the wrist voluntarily moves, in comparison to the healthy group. The results of these pairwise comparisons imply that coupling of the wrist joint with the shoulder and elbow joints is relatively stronger in the stroke group than in the healthy group. Pairwise comparisons separating movement directions show that the elbow joint in the stroke group involuntarily move greater when the wrist voluntarily moves, during inward ( $p$ $=0.036)$ and outward $(\mathrm{p}=0.024)$ movements. Fig. 3(b) presents significant differences between groups during inward and outward movements, respectively. One-tailed t-tests report that all ratios of joint motion are significantly lower than $1(\mathrm{p}<0.05)$, suggesting that all joints show a greater extent of voluntary motion versus involuntary motion produced by the other joints.

MANOVA reports no main effect of group in ratios for muscle activation coupling $(\mathrm{p}>0.6)$. Meanwhile, the stroke group shows significant greater ratios for the PD muscle for the cases of the Bi muscle involved $(p=0.031)$ and the Tri muscle involved $(p=0.012)$. The ratios for FCR for the case of the Tri muscle involved $(\mathrm{p}=0.031)$ and Tri for the case of the ECR muscle involved $(\mathrm{p}=0.016)$ are significantly different between the groups (see Fig. 3(b)). Marginally significant difference occurs in Tri $(\mathrm{p}=0.078)$ and FCR $(\mathrm{p}=0.095)$ when paired with voluntary activation of $\mathrm{AD}$, Tri $(\mathrm{p}=0.082)$ and FCR $(\mathrm{p}=0.095)$ when paired with voluntary activation of PD, FCR $(\mathrm{p}=$ $0.085)$ when paired with voluntary activation of $\mathrm{Bi}$, and Tri $(\mathrm{p}=0.059)$ when paired with voluntary activation of FCR.

One-tailed t-tests revealed that ratios for all cases others than three cases are significantly lower than 1 in the stroke group $(\mathrm{p}<0.05)$ : FCR when paired with Bi and Tri, and ECR when paired with Tri. The results indicate that stroke-impaired FCR and ECR involuntarily activate when Bi or Tri voluntarily activates in the same or a greater level as activation of FCR and ECR when they voluntarily activate.

\section{Results on stroke participants with severe distal joint impairment}


226

227

228

229

230

231

232

233

234

235

236

237

238

239

240

241

242

243

244

245

246

247

248

249

250

251

252

253

254

Fig. 4 shows the results of the correlation analyses. The UEFM score is significantly correlated with the angular displacement of the wrist joint during voluntary extension $(p=0.006)$ and the active range of motion (AROM) of the wrist $(\mathrm{p}=0.005)$. Based on the severity of distal joint impairment, we divide participants with stroke into two groups (group A and group B) to reveal the features of stroke-caused coupling based on the severity of corticospinal system damage. The stroke group A consists of 6 participants while the stroke group B consists of 8 participants (refer to Fig. 4). There are significant differences in wrist extension $(p=0.0001)$ and AROM $(p=0.0001)$ between the two groups.

MANOVA reveals a significant main effect of group for joint motion coupling $(F(6,66)=5.477$, $p<0.0001$, Wilk's $\Lambda=0.251$, partial $\left.\eta^{2}=0.499\right)$. No effects of movement direction $(p>0.9)$ and interaction $(\mathrm{p}>0.9)$ are observed, indicating no significant differences in the extent of motion coupling between inward movements and outward movements (see Fig. 5(a)). Pairwise comparisons separating movement directions between groups report that the wrist joint in the stroke group A involuntarily moves significantly greater when the shoulder voluntarily moves inward as well as when the shoulder voluntarily moves outward, in comparison to the stroke group $\mathrm{B}(\mathrm{p}<0.05)$ and the healthy group $(\mathrm{p}<0.05)$. The elbow joint in the stroke group A also involuntarily moves significantly greater when the wrist voluntarily moves inward in comparison to the stroke group $B(p=0.037)$ and the healthy group $(p=0.003)$. When the wrist voluntarily moves outward, the extent of involuntary elbow movement in the stroke group A is greater than the healthy group $(\mathrm{p}=0.006)$.

MANOVA reveals a marginally significant main effect of group for muscle activation coupling $\left(F(16,24)=1.919, p=0.090\right.$, Wilk's $\Lambda=0.066$, partial $\left.\eta^{2}=0.742\right)$. Fig. $5(b)$ presents the results of pairwise comparisons. Pairwise comparisons between groups report that the wrist extensor in the stroke group A involuntarily activates significantly greater with AD, PD or Tri voluntarily activating, in comparison to the stroke group B $(p<0.05)$ and the healthy group $(p<0.05)$. Marginally significant differences are exhibited between the stroke group A and healthy group, including the cases of involuntary activation of PD when Bi $(p=0.079)$ or Tri $(p=0.075)$ voluntarily activates. We note that one-tailed t-tests report that all ratios regarding $\mathrm{Bi}$, FCR and ECR in the stroke group A do not reach a significant difference from $1(\mathrm{p}>0.05)$, implying that 
the extent of involuntary activation of those muscles when another muscle voluntarily activates is not less than that of their voluntary activation.

\section{DISCUSSION}

Joint motion coupling - muscle activation coupling - is thought to be one of the main sources of abnormal coordination of movement following stroke. Joint coupling — muscle coupling — would indicate the cortical areas and/or neural pathways pertaining to each joint/muscle are interfered with one another following neural reorganization after stroke. We questioned whether strokecaused neural reorganization brings about directionality of coupling between two joints - two muscles - of the upper limb.

Our experiment results show that abnormal coupling in the upper extremity following stroke is mainly pronounced between the shoulder and wrist joints, between the elbow and wrist joints, and between their corresponding muscles, although marginal significances of $p<0.1$ also exist. Note that abnormal coupling occurs between nonadjacent joints as well as between adjacent joints. In particular, the finding of abnormal coupling between the shoulder and wrist joints is in accordance with previous studies(Seo, Rymer and Kamper, 2009; McPherson and Dewald, 2019; Senesh, Barragan and Reinkensmeyer, 2020). The so-called flexion synergy and extension synergy work in the affected individuals and involve abnormal co-activation of shoulder abductor and adductor with distal limb flexor and extensor, respectively(Beer, Dewald and Rymer, 2000; Kamper and Rymer, 2001; Wright, Rymer and Slutzky, 2014). In both the shoulder and elbow, the extent of motion generated when these joints are the target joint are clearly differentiable from the case when these joints are the non-target joint for the stroke and healthy groups, regardless of whether participants move these joints inward or outward (see Fig. 3). We could find the primary reason of it from the effect of gravity on movements of the shoulder and elbow. In our study, participants made movements in the horizontal plane; the effect of gravity was removed. The constraint of movements in the horizontal plane might lead to an increase in the range of motion at the elbow for the stroke group, which can be linked with the comparable results of the SHL-ELB pair in the stroke group to that in the healthy group(Ada, Canning and Low, 2003; Beer et al., 2007). 
We note that abnormal coupling in the stroke group is directional. Mainly, the wrist flexor in the stroke group tends to subordinately activate when the shoulder muscles voluntarily activate, whereas when FCR voluntarily activates, $\mathrm{AD}$ and PD are not subordinately activated, at least, to a similar degree to when AD or PD volitionally activates, although the statistical analysis merely reaches marginal significances $(\mathrm{p}<0.1)$. These results would suggest that the cortical sources and/or neural pathways of FCR and ECR are paired with those of AD and PD, and that separate allocations in the corticospinal system exist for $\mathrm{AD}$ and $\mathrm{PD}$ that are not disturbed by those for FCR and ECR.

Another observation regarding directional coupling in the stroke group versus the healthy group is in the case of the ELB-WRS pair. We see that FCR marginally significantly co-activates with $\mathrm{Bi}$ activating in the stroke group in comparison with the healthy group $(\mathrm{p}<0.05)$. Co-activation between $\mathrm{Bi}$ and FCR probably results from the flexion synergy occurring with the loss of the corticospinal system input, leading to an increased use of alternative motor pathways when participants move their elbow inward(Herbert, Davidson and Buford, 2010). Meanwhile, the extents of Bi co-activating with FCR activating in both groups are not significantly different ( $p>$ 0.2). These results provide evidence of the directionality of abnormal coupling between $\mathrm{Bi}$ and FCR.

The phenomenon that we observe ratios not significantly less than 1 in the biceps and triceps across healthy participants is due partially to an effort of stabilization of the forearm through an anticipatory postural adjustment(Kim, 2019). Interestingly, the stroke group shows a ratio less than 1 in the triceps muscle, indicating impaired anticipatory control in individuals with stroke in suppressing interaction torques that exist in a multi-joint arm movement(Bradnam, Stinear and Byblow, 2013; Capaday et al., 2013).

A questioned arose here: does directional abnormal coupling originate from the severity gradient of motor deficits of proximal joints to distal joints? Fig. 4 shows correlations of the UEFM score with the maximum angular displacements during voluntary flexion and extension of the shoulder, elbow and wrist, respectively. These results suggest that stroke participants with the UEFM score below 26 show severe impairment at the wrist joint, except for one participant with the UEFM score 10. Those participants, called the stroke group A, have difficulty in extending the wrist joint and a reduced active range of motion at this joint. This phenomenon may result from that the stroke 
group A uses the RST instead of the CST. Use of the RST is known to lead to a notable gradient of motor deficits of proximal joints to distal joints(Turton et al., 1996). The stroke group A is reasonably assumed to primarily employ the RST while the stroke group B, who shows less severe impairment at the proximal joint, is reasonably assumed to primarily employ the CST, which is in accordance with the theory presented by Senesh et al(Senesh, Barragan and Reinkensmeyer, 2020),

Fig. 5 exhibits the results of joint motion coupling and muscle activation coupling in the stroke group A versus the stroke group B and healthy group. The results with the separated stroke groups reveal a greater extent of abnormal motion coupling between the shoulder and wrist joints and between the elbow and wrist is found in the stroke group A in comparison to the other groups ( $p$ $<0.05$ ). We note that motion coupling between the shoulder and wrist joints is directional; the coupling occurs only when the shoulder voluntarily moves, not when the wrist voluntarily moves. Also motion coupling between the elbow and wrist joints could be regarded directional; the extent of involuntary motion of the elbow when the wrist voluntarily moves is significantly less than that of its voluntary motion, whereas the extent of involuntary motion of the wrist when the elbow voluntarily moves is not significantly less than that of its voluntary motion (refer to Fig. 5).

The results of muscle activation coupling in the stroke group A show a greater extent of abnormal involuntary activation of ECR when AD, PD, FCR, or ECR voluntarily activates in comparison to the other groups $(\mathrm{p}<0.05)$. It could be speculated that ECR involuntarily co-activates with other muscles due mainly to the RST that excites ipsilateral extensors as well as ipsilateral flexors (significant differences on FCR in this separated stroke group are not made due to the small sample size, while (marginally) significant differences on FCR in the whole stroke group are made)(Gahery, 1987; Takahashi and Reinkensmeyer, 2003). Meanwhile, the stroke group B shows ratios of similar levels regarding involuntary activation of ECR as the healthy group, justifying the assumption that this group employs the CST that facilities controllability of individual muscles as in the healthy group(Baker, 2011).

The directional neural couplings we observed at least in this study probably originate from the diversity of cortical sources or descending motor pathways from the cortex to the muscles associated with a certain joint(McMorland, Runnalls and Byblow, 2015). The contralateral CST is the main conveyor of voluntary neural drive to a healthy upper extremity. While some CST connections exist for cortically derived synergies that control neuron pools for proximal joints, 
some connections convey low-dimensional motor commands and innervate synergy-forming spinal modules that are connected to multiple motor neuron pools. While interhemispheric pathways to the contralateral motor cortex linking to the brainstem and cortico-reticulopropriospinal pathway that activate multiple proximal motor neuron pools, direct CST fibers from the cortical sources to distal motor neuron pools are present. A stroke-caused lesion leads activation at a certain region on the cortex to activation of adjacent regions, which innervates all motor neuron pools in the representations at the regions(Wickens, Hyland and Anson, 1994; Capaday et al., 2013). Or a lesion disrupts and entangles neural pathways that carry motor commands from the cortex, which also innervates all motor neuron pools linked with the pathways(Bradnam, Stinear and Byblow, 2013). However, multiple cortical sources and multiple pathways exist for a certain motor neuron pool. Even if a source and/or pathway of the motor neuron pool for the joint to move is interfered with that for other joints, there could be another source/pathway intact to move the target joint. This possibility leads to unidirectional couplings. Perhaps the existence of a direct CST connection to the motor neuron pool for the wrist joint could explain that the wrist joint moves in an involuntary mode when a more proximal joint voluntarily moves, whereas the more proximal joint does not move in an involuntary mode when the wrist joint voluntarily moves.

For the stroke group A who is assumed to be under use of the RST, the directionality of abnormal coupling could be attributed to the severely damaged distal joint (i.e. wrist joint). While this group moves the shoulder joint (and also the elbow joint) in the similar level to the stroke group B, it has a significantly decreased ability to move the wrist joint as seen in Fig. 4. The decreased ability to move the wrist joint leads to the phenomena that the degree of wrist muscles activating in an involuntary manner through the flexion or extension synergies when other muscles voluntarily activate is of a greater or the similar level to that activating when the wrist voluntarily moves. However we should note that muscles for proximal joints (i.e. wrist and finger joints) in stroke individuals can activate when they intend to move(Chae et al., 2002; Seo, Rymer and Kamper, 2009), even in a timely manner [unpublished]. Even if the stroke group A is assumed to be unable to use a direct CST for the wrist joint, our study demonstrates that activation of wrist muscles does not lead to involuntary activation of shoulder or elbow muscles, which provides evidence of directionality of stroke-caused coupling. 
373 The biggest limitation of this study is the small same size, while stroke is caused with large 374 variability in lesioned regions in the brain. This study should be validated with fMRI or 375 electroencephalography (EEG) technologies to identify neural sources. However, we raised the 376 possibility that couplings from the flexion synergy or extension synergy can be directional. This 377 knowledge would be expected to present a significant step towards personalized, mechanism378 oriented therapeutic strategies.

\section{Sources of Funding}

381 This research study was supported by the National Institute on Disability, Independent Living, 382 and Rehabilitation Research (Grant \# 90DP0099). The first author was also supported by the 383 University of Maryland Baltimore Institute for Clinical and Translational Research (ICTR) which 384 is funded in part by Grant Number TL1 TR003100 from the National Center for Advancing 385 Translational Sciences (NCATS) a component of the National Institutes of Health (NIH), and NIH 386 Roadmap for Medical Research.

387

\section{REFERENCES}

389 Ada, L., Canning, C. G. and Low, S. L. (2003) 'Stroke patients have selective muscle weakness in shortened range', Brain, 126(3), pp. 724-731. doi: 10.1093/brain/awg066. Baker, S. N. (2011) 'The primate reticulospinal tract, hand function and functional recovery', 392 Journal of Physiology, 589(23), pp. 5603-5612. doi: 10.1113/jphysiol.2011.215160. 395 doi: 10.1080/10749357.2021.1894660.

396 Beebe, J. A. and Lang, C. E. (2008) 'Absence of a proximal to distal gradient of motor deficits in 397 the upper extremity early after stroke', Clinical Neurophysiology, 119(9), pp. 2074-2085. doi: 398 10.1016/j.clinph.2008.04.293.

399 Beer, R. F. et al. (2007) 'Impact of gravity loading on post-stroke reaching and its relationship to 
400

401

402

403

404

405

406

407

408

409

410

411

412

413

414

415

416

417

418

419

420

421

422

423

424

425

426

427

weakness', Muscle and Nerve, 36(2), pp. 242-250. doi: 10.1002/mus.20817.

Beer, R. F., Dewald, J. P. A. and Rymer, W. Z. (2000) 'Deficits in the coordination of multijoint arm movements in patients with hemiparesis: evidence for disturbed control of limb dynamics', Experimental Brain Research, 131(3), pp. 305-319. doi: 10.1007/s002219900275.

Bradnam, L. V., Stinear, C. M. and Byblow, W. D. (2013) 'Ipsilateral motor pathways after stroke: Implications for noninvasive brain stimulation', Frontiers in Human Neuroscience, 7(APR 2013), pp. 1-8. doi: 10.3389/fnhum.2013.00184.

Capaday, C. et al. (2013) 'On the functional organization and operational principles of the motor cortex', Frontiers in Neural Circuits, 7(MAR), pp. 1-15. doi: 10.3389/fncir.2013.00066.

Chae, J. et al. (2002) 'Delay in initiation and termination of muscle contraction, motor impairment, and physical disability in upper limb hemiparesis', Muscle and Nerve, 25(4), pp. 568-575. doi: 10.1002/mus.10061.

Cheung, V. C. K., D’Avella, A. and Bizzi, E. (2009) 'Adjustments of motor pattern for load compensation via modulated activations of muscle synergies during natural behaviors', Journal of Neurophysiology. American Physiological Society, 101(3), pp. 1235-1257. doi: 10.1152/jn.01387.2007.

Cirstea, M. C. and Levin, M. F. (2000) 'Compensatory strategies for reaching in stroke', Brain. Oxford University Press, 123(5), pp. 940-953. doi: 10.1093/brain/123.5.940.

Colebatch, J. G. and Gandevia, S. C. (1989) 'The distribution of muscular weakness in upper motor neuron lesions affecting the arm', Brain, 112(3), pp. 749-763. doi: 10.1093/brain/112.3.749.

Ellis, M. D. et al. (2016) 'Robotic quantification of upper extremity loss of independent joint control or flexion synergy in individuals with hemiparetic stroke: a review of paradigms addressing the effects of shoulder abduction loading', Journal of NeuroEngineering and Rehabilitation. Journal of NeuroEngineering and Rehabilitation, 13(1), pp. 1-11. doi: 10.1186/s12984-016-0203-0.

Fetz, E. E. and Cheney, P. D. (1980) 'Postspike facilitation of forelimb muscle activity by primate corticomotoneuronal cells', Journal of Neurophysiology, 44(4), pp. 751-772. doi: 
429 Gahery, Y. (1987) 'Associated movements, postural adjustments and synergie: Some comments 430 about the history and significance of three motor concepts', Archives Italiennes de Biologie, 431 125(4), pp. 345-360. doi: 10.4449/aib.v125i4.1001.

432 Herbert, W. J., Davidson, A. G. and Buford, J. A. (2010) 'Measuring the motor output of the 433 pontomedullary reticular formation in the monkey: Do stimulus-triggered averaging and stimulus 434 trains produce comparable results in the upper limbs?', Experimental Brain Research, 203(2), 435 pp. 271-283. doi: 10.1007/s00221-010-2231-5.

436 Kamper, D. G. and Rymer, W. Z. (2001) 'Impairment of voluntary control of finger motion 437 following stroke: Role of inappropriate muscle coactivation', Muscle and Nerve, pp. 673-681. 438 doi: 10.1002/mus.1054.

439 Kim, D. (2019) 'A computational scheme for internal models not requiring precise system 440 parameters', PLOS ONE. doi: 10.1371/journal.pone.0210616.

441 McMorland, A. J. C., Runnalls, K. D. and Byblow, W. D. (2015) 'A neuroanatomical framework 442 for upper limb synergies after stroke', Frontiers in Human Neuroscience, 9(FEB), pp. 1-6. doi: $443 \quad 10.3389 /$ fnhum.2015.00082.

444 McPherson, L. M. and Dewald, J. P. A. (2019) 'Differences between flexion and extension 445 synergy-driven coupling at the elbow, wrist, and fingers of individuals with chronic hemiparetic 446 stroke', Clinical Neurophysiology. International Federation of Clinical Neurophysiology, 130(4), 447 pp. 454-468. doi: 10.1016/j.clinph.2019.01.010.

448 Owen, M., Ingo, C. and Dewald, J. P. A. (2017) 'Upper extremity motor impairments and 449 microstructural changes in bulbospinal pathways in chronic hemiparetic stroke', Frontiers in 450 Neurology, 8(JUN). doi: 10.3389/fneur.2017.00257.

451 Roh, J. et al. (2013) 'Alterations in upper limb muscle synergy structure in chronic stroke 452 survivors', Journal of Neurophysiology. American Physiological Society Bethesda, MD, 109(3), 453 pp. 768-781. doi: 10.1152/jn.00670.2012.

454 Schieber, M. H. (2001) 'Constraints on somatotopic organization in the primary motor cortex', 455 Journal of Neurophysiology, 86(5), pp. 2125-2143. doi: 10.1152/jn.2001.86.5.2125. 
456 Senesh, M. R., Barragan, K. and Reinkensmeyer, D. J. (2020) 'Rudimentary Dexterity

457 Corresponds With Reduced Ability to Move in Synergy After Stroke: Evidence of Competition

458 Between Corticoreticulospinal and Corticospinal Tracts?', Neurorehabilitation and Neural

459 Repair, 34(10), pp. 904-914. doi: 10.1177/1545968320943582.

460 Seo, N. J., Rymer, W. Z. and Kamper, D. G. (2009) 'Delays in grip initiation and termination in

461 persons with stroke: Effects of arm support and active muscle stretch exercise', Journal of

462 Neurophysiology, 101(6), pp. 3108-3115. doi: 10.1152/jn.91108.2008.

463 Takahashi, C. D. and Reinkensmeyer, D. J. (2003) 'Hemiparetic stroke impairs anticipatory

464 control of arm movement', Experimental Brain Research, 149(2), pp. 131-140. doi:

465 10.1007/s00221-002-1340-1.

466 Turton, A. et al. (1996) 'Contralateral and ipsilateral EMG responses to transcranial magnetic

467 stimulation during recovery of arm and hand function after stroke', Electroencephalography and

468 Clinical Neurophysiology - Electromyography and Motor Control, 101(4), pp. 316-328. doi:

469 10.1016/0924-980X(96)95560-5.

470 Wickens, J., Hyland, B. and Anson, G. (1994) 'Cortical cell assemblies: A possible mechanism

471 for motor programs', Journal of Motor Behavior, 26(2), pp. 66-82. doi:

$47210.1080 / 00222895.1994 .9941663$.

473 Wilkins, K. B. et al. (2017) 'Neural plasticity in moderate to severe chronic stroke following a

474 device-assisted task-specific arm/hand intervention', Frontiers in Neurology, 8(JUN), pp. 1-11.

475 doi: 10.3389/fneur.2017.00284.

476 Wright, Z. A., Rymer, W. Z. and Slutzky, M. W. (2014) 'Reducing abnormal muscle

477 coactivation after stroke using a myoelectric-computer interface: A pilot study',

478 Neurorehabilitation and Neural Repair. doi: 10.1177/1545968313517751.

479 Yang, Y. et al. (2018) 'Unveiling neural coupling within the sensorimotor system: directionality

480 and nonlinearity', European Journal of Neuroscience, 48(7), pp. 2407-2415. doi:

481 10.1111/ejn.13692.

482 
Wrist In

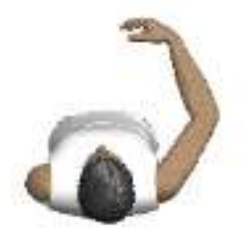

Elbow In

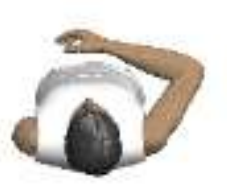

Shoulder In

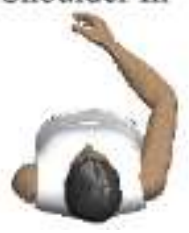

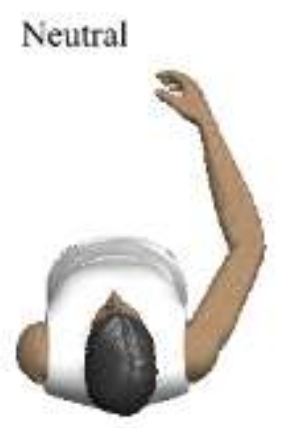

488

489

490

491

Fig. 1. Experimental procedure. Participants move each of the shoulder, elbow and wrist joints back and forth from the neutral posture, respectively, with the other joints resting. 


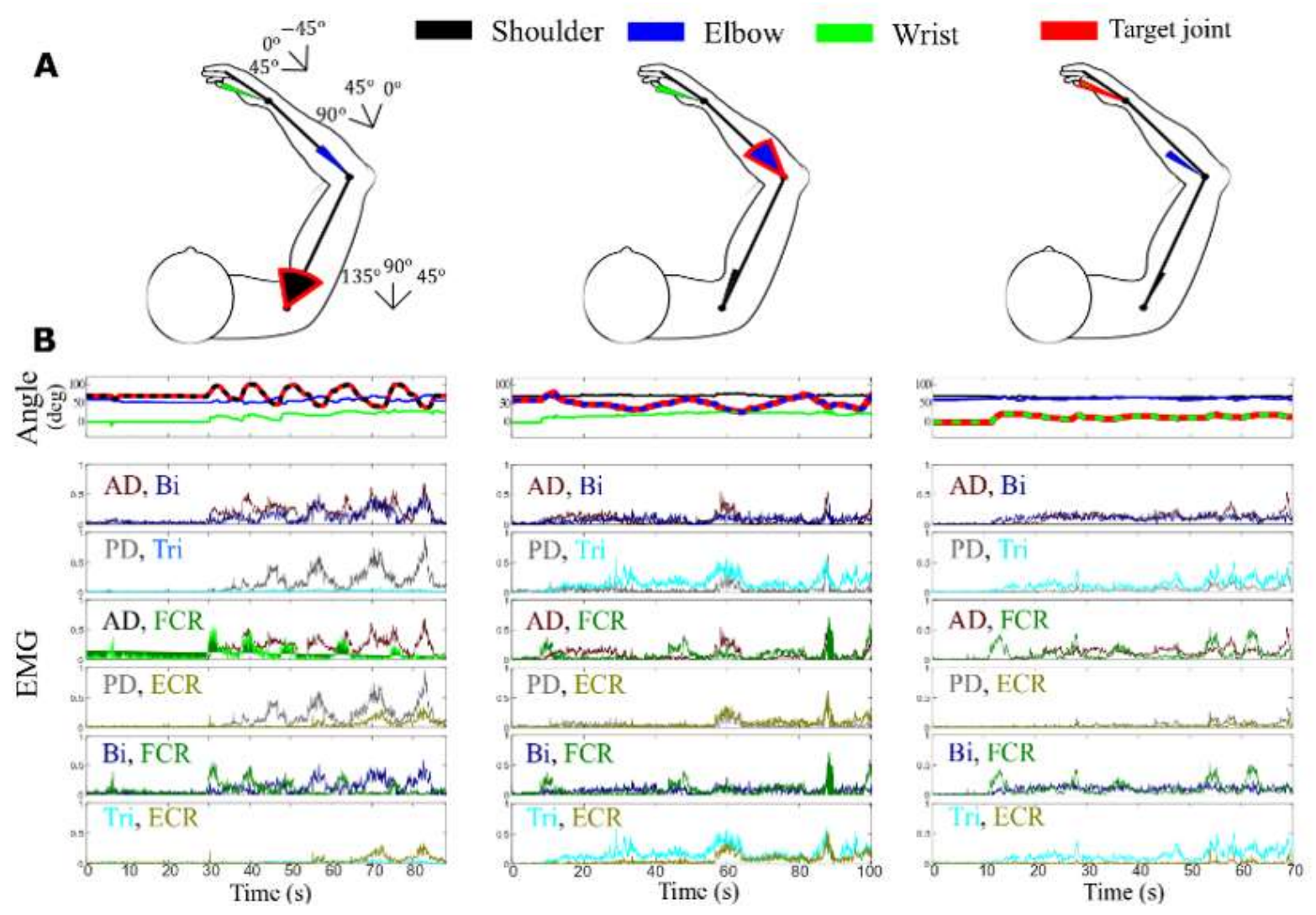

494 Fig. 2. Results of a representative individual post stroke. (A) Schematics of movement during 495 each session show the active range of motion of the target joint (wrapped with red) and the ranges 496 of motion of the non-target joints that move in a non-volitional mode. (B) Plots display the 497 trajectories of the shoulder, elbow and wrist angles and the EMG LEs of AD, PD, Bi, Tri, FCR, 498 and ECR muscles during each session, respectively. 


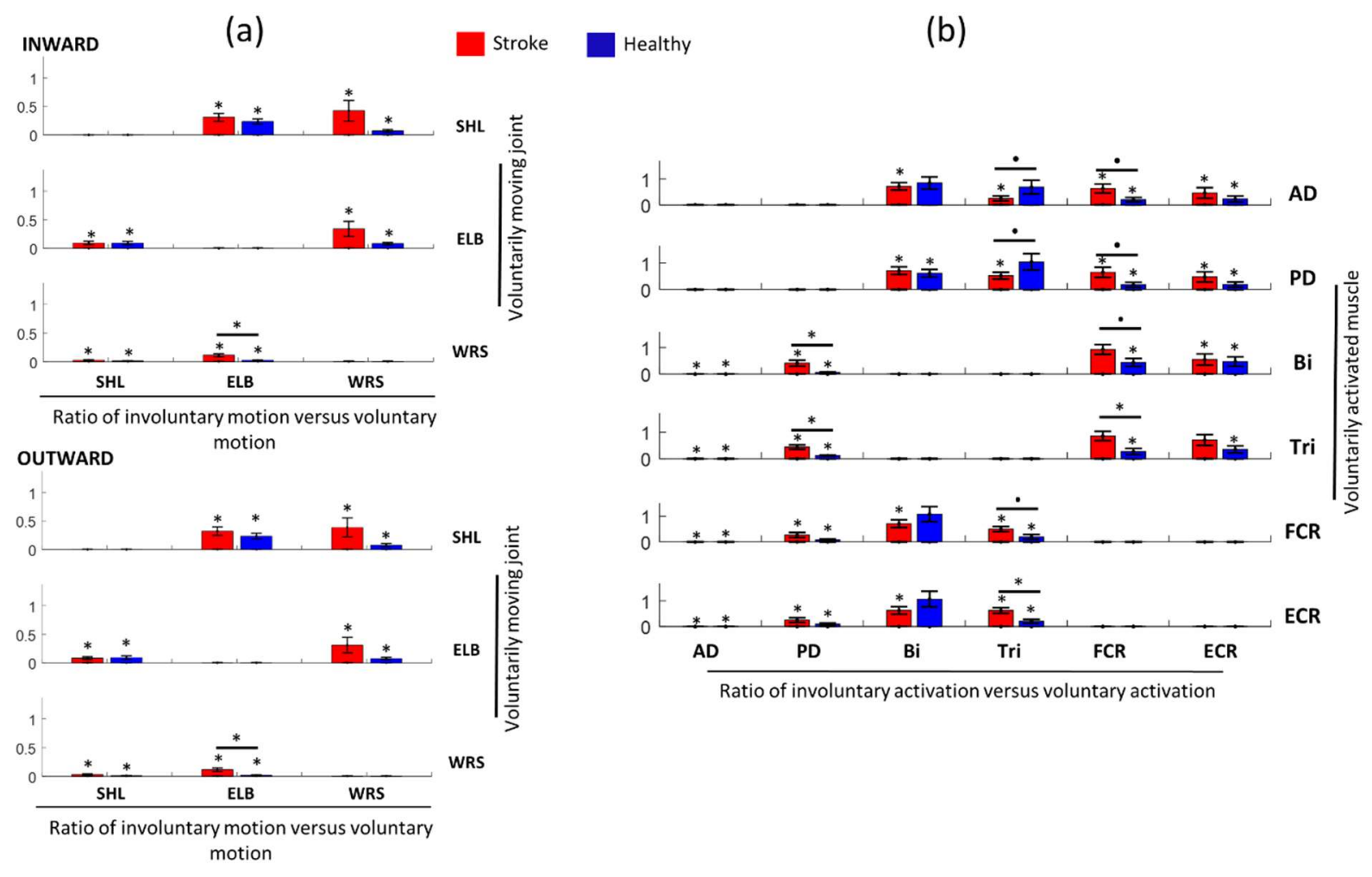

Fig. 3. Results of (a) joint motion coupling and (b) muscle activation coupling in the whole

501 stroke participants versus healthy participants. For joint motion coupling, ratios indicate the

502 extent of involuntary motion (by another joint motion) versus that of voluntary motion at a joint

503 of interest. For muscle activation coupling, ratios indicate the extent of involuntary activation (by

504 another muscle activation) versus that of voluntary activation at a muscle of interest. Error bars

505 are \pm 1 standard error of the mean. Asterisks indicate a significant difference $(\mathrm{p}<0.05)$ while dots 506 indicate a marginally significant difference $(\mathrm{p}<0.1)$.

507 


\section{Flexion}
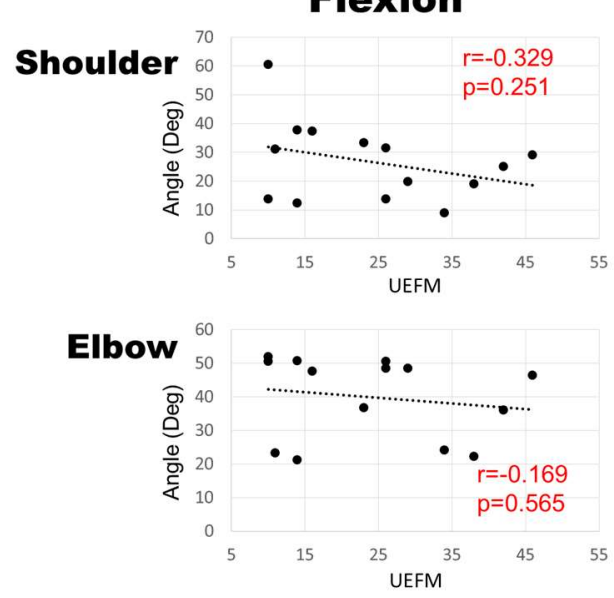

Wrist

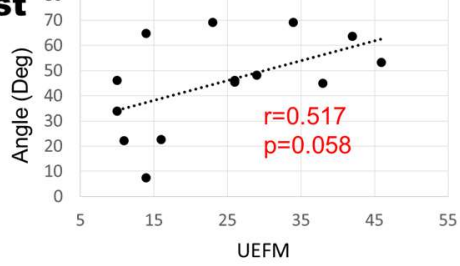

Extension
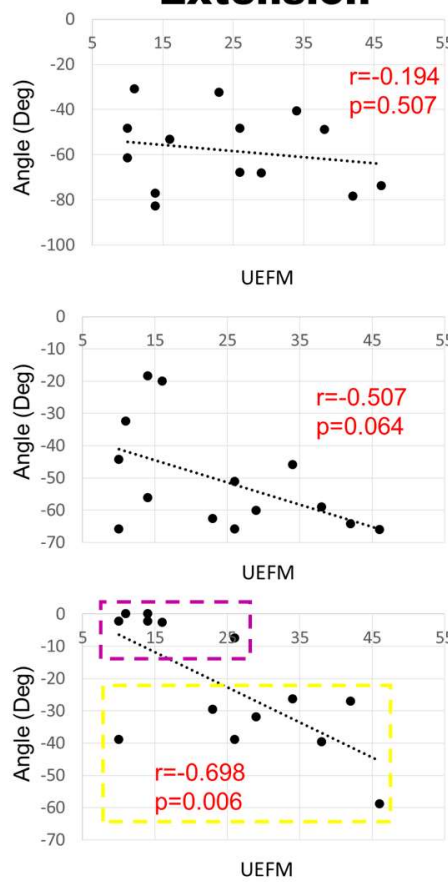

Active ROM
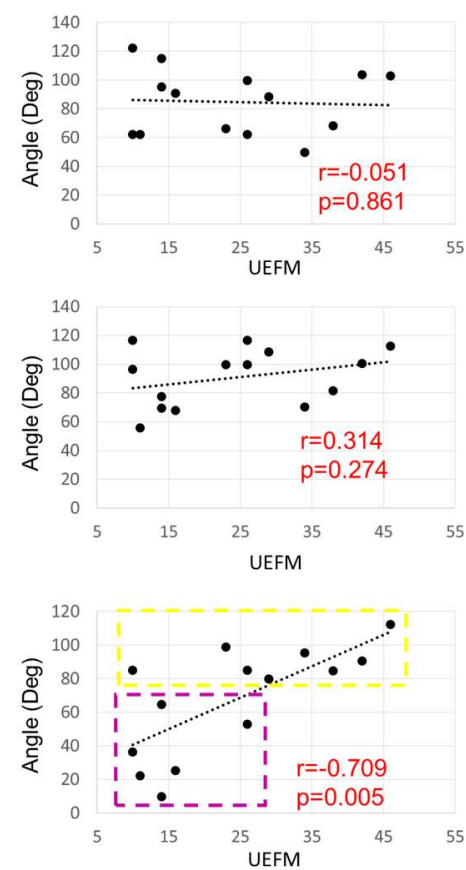

Fig. 4. Plots of the maximum angular displacements during voluntary flexion and extension

511 of the shoulder, elbow and wrist, versus the total UEFM score. Trend lines are presented with

512 Pearson correlation coefficients and p-values. Based on the results of the correlation analyses, we

513 divide stroke participants into two groups: one group with severe distal joint impairment (Stroke

514 A, pink dashed box) and the other group consisting of the remaining stroke participants (Stroke B,

515 yellow dashed box). Stroke group A is assumed to primarily use the RST, while stroke group B is

516 assumed to primarily use the CST based on the theory presented by a study(Senesh, Barragan and

517 Reinkensmeyer, 2020). 


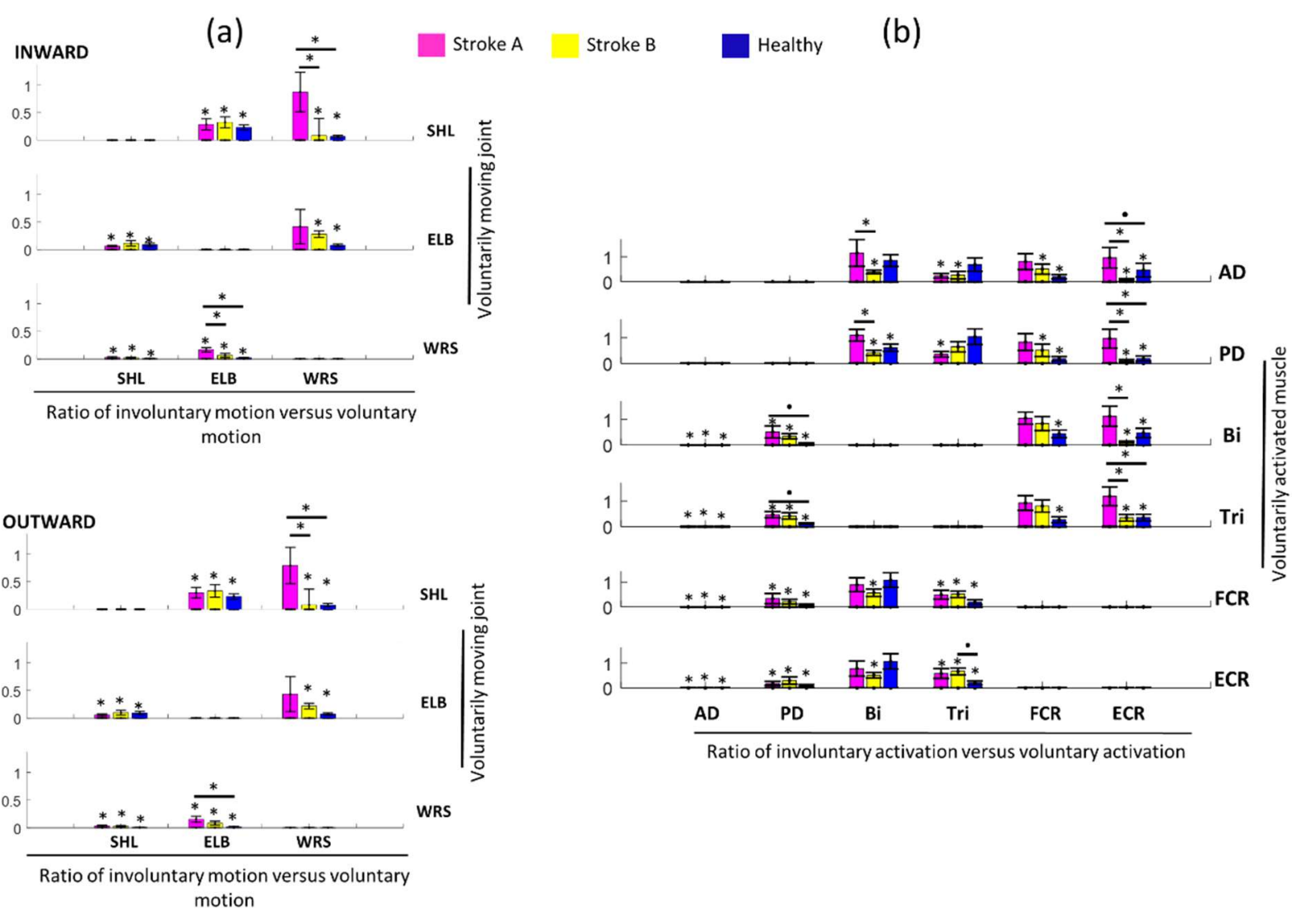

519 Fig. 5. Results of (a) joint motion coupling and (b) muscle activation coupling in stroke participants with severe dismal joint impairment (Stroke A) versus the remaining stroke

521 participants (Stroke B) and healthy participants. For joint motion coupling, ratios indicate the

522 extent of involuntary motion (by another joint motion) versus that of voluntary motion at a joint

523 of interest. For muscle activation coupling, ratios indicate the extent of involuntary activation (by

524 another muscle activation) versus that of voluntary activation at a muscle of interest. Error bars

525 are \pm 1 standard error of the mean. Asterisks indicate a significant difference $(\mathrm{p}<0.05)$ while dots 526 indicate a marginally significant difference $(\mathrm{p}<0.1)$. 